\title{
Performance improvement in Brillouin-based simultaneous strain and temperature sensors employing pulse coding in coherent detection schemes
}

\author{
Marcelo A. Soto, Gabriele Bolognini, Fabrizio Di Pasquale \\ Scuola Superiore Sant'Anna, via G. Moruzzi 1, 56124 Pisa, Italy.e-mail: gabriele.bolognini@sssup.it
}

\begin{abstract}
We have experimentally characterized the benefits of pulse coding in coherent-detection Brillouin-based hybrid sensors, achieving $15 \mathrm{~dB}$ improvement in two-way sensing distance at same resolution in simultaneous strain and temperature sensing.

(C)2009 Optical Society of America

OCIS codes: (060. 2370) Fiber optics sensors, (290.5830) Scattering, Brillouin.
\end{abstract}

\section{Introduction}

Distributed optical fiber sensors are recently attracting a growing interest both from research and industrial sectors [1], due to the wide range of applications they can be exploited for - from environmental or structuralhealth monitoring to fire-detection and security systems - and also thanks to their unique distributed measurement capability. Distributed sensors most commonly exploit scattering effects, such as Raman or Brillouin scattering, within time-domain measurement schemes [2], allowing one to detect fiber-related properties like temperature and strain. In particular, Raman-based sensors are sensitive to temperature only, while Brillouin-based sensors are inherently sensitive to both strain and temperature. Brillouin-based systems can employ spontaneous scattering (SpBS) as well as stimulated scattering (SBS); while the latter technique is characterized by higher accuracy, requiring complex schemes and access to both fiber ends, SpBS-based sensors offer a simpler implementation, accessing to one fiber-end only, and providing a practical and cost-effective solution in many applications [1]. In order to distinguish temperature from strain within the same optical fiber, Brillouin-based sensors require to measure both Brillouin intensity and frequency shift, which can be easily implemented through coherent-detection systems [3]. In such schemes, the main issue limiting the attainable resolution comes from the noise on the intensity measurement [3]. In this work, hence, we apply optical pulse coding to spontaneous Brillouin-based sensors for simultaneous temperature and strain sensing based on coherent detection of both SpBS intensity and frequency shift (BFS), resulting in a significant enhancement of temperature-strain sensing range thanks to the combined used of coding and coherent detection.

\section{Theory}

In SpBS-based sensors, simultaneous hybrid sensing is achieved thanks to the cross-sensitivity of both BFS and intensity to fiber temperature and strain. This feature allows for sensing both physical variables simultaneously by measuring both BFS and SpBS power traces. The fiber-loss-independent Landau-Placzek ratio (LPR) [4] (i.e. the ratio of the Rayleigh scattering power to the SpBS power), or its inverse (i.e. the normalized SpBS power), are generally used instead of the loss-dependent SpBS power.

The changes in BFS $\left(\Delta v_{B}\right)$ and normalized SpBS power $\left(\Delta P_{B}\right)$ due to strain $(\Delta \varepsilon)$ and temperature $(\Delta T)$ variations can be represented through a matrix equation [5], which can be suitably inverted to provide the strain and temperature estimation. The noise, defined as the $r m s$ error in BFS $\left(\delta v_{B}\right)$ and normalized SpBS power $\left(\delta P_{B}\right)$ measurements, will then affect the final accuracy of estimated $\Delta T$ and $\Delta \varepsilon$. The former matrix equation and the respective $r m s$ error in temperature $(\delta T)$ and strain $(\delta \varepsilon)$ estimation are reported below:

$$
\left[\begin{array}{c}
\Delta v_{B} \\
\Delta P_{B}
\end{array}\right]=\left[\begin{array}{ll}
C_{v_{B} \varepsilon} & C_{v_{B} T} \\
C_{P_{B} \varepsilon} & C_{P_{B} T}
\end{array}\right]\left[\begin{array}{c}
\Delta \varepsilon \\
\Delta T
\end{array}\right] \quad(1), \quad \delta \varepsilon=\frac{\left|C_{P_{B} T}\right| \delta v_{B}+\left|C_{v_{B} T}\right| \delta P_{B}}{\left|C_{v_{B} \varepsilon} C_{P_{B} T}-C_{P_{B} \varepsilon} C_{v_{B}}\right|}, \delta T=\frac{\left|C_{P_{B} \varepsilon}\right| \delta v_{B}+\left|C_{v_{B} \varepsilon}\right| \delta P_{B}}{\mid C_{v_{B} \varepsilon} C_{P_{B} T}-C_{P_{B} \varepsilon} C_{v_{B}} T}
$$

where $\mathrm{C}_{\mathrm{VB} \varepsilon}, \mathrm{C}_{\mathrm{vBT}}, \mathrm{C}_{\mathrm{PB} \varepsilon}$ and $\mathrm{C}_{\mathrm{PBT}}$ are the strain and temperature coefficients for BFS and SpBS power [5].

We recently demonstrated that optical coding, namely Simplex coding [6], can be effectively employed in direct-detection LPR-based Brillouin distributed temperature sensors (BDTS), providing significant temperature resolution improvement due to electrical signal-to-noise ratio (SNR) enhancement with respect to conventional single-pulse schemes. This enhancement is expressed by the coding gain $G_{C O D}=(L+1) /(2 \sqrt{ } L)$, which depends on code length $L$. On the other hand, in coherent-detection schemes, the SpBS signal is mixed with an optical local oscillator (OLO) before photodetection, allowing for higher sensitivity and dynamic range in comparison to direct-detection schemes. It is worth noting that the use of coherent-detection in conjunction with optical coding can in principle bring a two-fold benefit; since the expected dB-improvement provided by pulse coding (in the electrical domain trace) with coherent detection is doubled, compared to direct detection, thanks to the square- 


\section{OThU4.pdf}

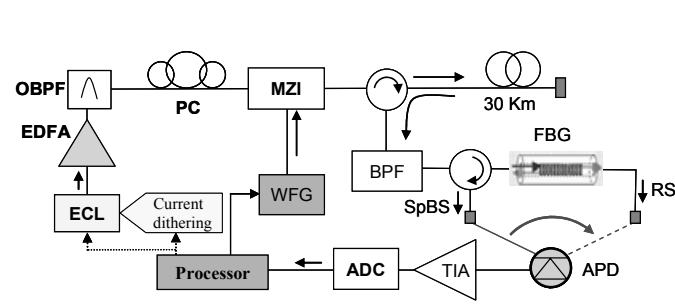

(a)

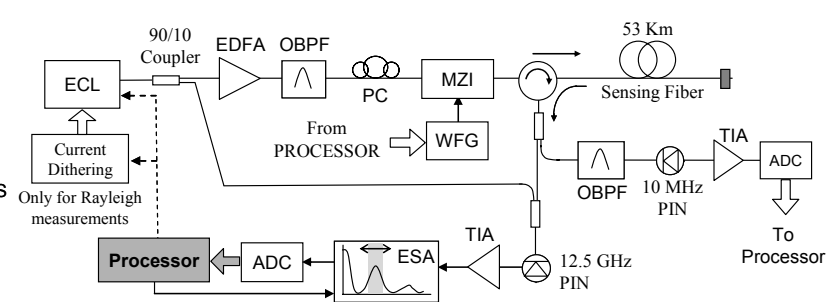

(b)

Fig. 1. Experimental set-up (a) BDTS based on direct detection (b) Hybrid Brillouin sensor based on coherent detection.

root dependence of the photocurrent, allowing for a doubled dB-benefit in optical budget and consequently in sensing range, with respect to pulse coding used in direct-detection schemes, as it will be shown in next sections.

\section{Experimental setup}

In this work two experimental setups have been implemented, one based on direct-detection scheme and a second one based on coherent detection. By measuring both SpBS power and Rayleigh signals, the benefits of pulse coding in coherent-detection LPR-based BDTS can be compare to the direct-detection case. Then, the second setup is employed to analyze the impact of pulse coding in simultaneous strain and temperature sensing.

Fig. 1a shows a LPR-based sensor implemented with a direct-detection receiver. The transmitter includes an external cavity laser (ECL, $200 \mathrm{kHz}$ linewidth) tuned at $1550.1 \mathrm{~nm}$, an EDFA, an optical band-pass filter (OBPF) to reduce ASE noise, a polarization-controller (PC) and a Mach-Zehnder modulator (MZI) controlled by a waveform generator (WFG) to intensity-modulate the $\mathrm{CW}$-light with either single pulses or 127-bit Simplexcoded pulses. The sensing fiber consists of $30 \mathrm{~km}$ dispersion-shifted fiber (DSF). The direct-detection receiver is based on a three-port optical circulator, a narrowband fiber Bragg grating (FBG, $6 \mathrm{GHz}$ bandwidth at antiStokes port) to separate the Anti-Stokes Brillouin signal from the Rayleigh component, an avalanche photodiode (APD, $80 \mathrm{MHz}$ ), a high-gain transimpedance amplifier (TIA) and an analog-digital converter (ADC).

The second experimental setup, shown in Fig $1 \mathrm{~b}$, includes a self-heterodyne receiver, which allows simultaneous SpBS intensity and BFS measurements. The light from an ECL, operating at $1555.0 \mathrm{~nm}$, is split into two parts; one part is used as OLO at the receiver and the second part is modulated as described in the previous setup. In this case, the sensing fiber consists of $53 \mathrm{~km}$ of DSF. At the receiver-side, a small portion of power is split out and sent to an OBPF, a $10 \mathrm{MHz}$ PIN photodiode and a TIA, in order to measure the necessary Rayleigh component when calculating the LPR or the normalized SpBS power. The Brillouin signal is then mixed with the OLO and then detected by a PIN photodiode followed by a TIA (12.5 GHz bandwidth). The OLO-Brillouin beat spectrum (at $\sim 11 \mathrm{GHz}$ ) is acquired using a $40 \mathrm{GHz}$ electric spectrum analyzer (ESA) operating in zero-span mode. To obtain the Brillouin scattering spectrum accurately, several OTDR traces are measured within the Brillouin band in $3 \mathrm{MHz}$ steps, followed by averaging and data processing. After averaging and fitting the spectrum with a Lorentzian curve, by using a least-squares estimation algorithm, the BFS evolution along the fiber is obtained. In both cases, a pulse duration of $400 \mathrm{~ns}$ has been used, allowing for $40 \mathrm{~m}$ spatial resolution; traces are averaged 180k times in case of single-pulses and 180k/127 times for each codeword in case of coding. Note that, when measuring the Rayleigh component to calculate LPR, coherent Rayleigh noise (CRN) needs to be reduced. In both experimental setups, $\mathrm{CRN}$ has been reduced down to $0.4 \%$ by using both laser current dithering and $\lambda$-averaging over $0.5 \mathrm{~nm}$.

\section{Impact of optical pulse coding in BDTS based on direct and coherent-detection schemes}

In order to compare the impact of optical pulse coding on the performance of LPR-based BDTS using coherentdetection and direct-detection schemes, the temperature resolution along the sensing fiber has been obtained for both cases. Fig. 2a shows the temperature resolution enhancement provided by coding technique when using the direct-detection scheme. The temperature resolution, $\sim 30 \mathrm{~K}$, achieved at $30 \mathrm{~km}$ using the single-pulsed BDTS is improved to $5.1 \mathrm{~K}$ when using 127-bit Simplex coding, allowing for $7.6 \mathrm{~dB}$ two-way fiber loss compensation (in agreement with the theoretical $G_{C O D}=7.54 \mathrm{~dB}$ ). Note that, the conventional BDTS offers a resolution of $5.1 \mathrm{~K}$ at a shorter distance of $\sim 12.5 \mathrm{~km}$, pointing out a sensing range enhancement of $\sim 17.5 \mathrm{~km}$ when using coding.

Fig. $2 \mathrm{~b}$ reports the temperature resolution obtained when implementing LPR-based BDTS using coherent detection (the SpBS power is now obtained by integrating the Brillouin scattering spectrum along the sensing fiber). A better accuracy in the measurements is achieved in this case, with respect to direct-detection schemes, mainly due to the amplification provided by the OLO to the Brillouin signal. A temperature resolution of $\sim 10 \mathrm{~K}$ is achieved in this case by the single-pulse BDTS at fiber-end ( $53 \mathrm{~km}$ distance), which is improved to $1.7 \mathrm{~K}$ by the use of coding. The experimental coding gain results to be $G_{C O D}=\sim 7.5 \mathrm{~dB}$, allowing for a significant electrical SNR improvement at the receiver. This also means that, in case the same resolution (i.e. SNR) is compared between single pulses and coding case, coding technique is particularly beneficial in conjunction with coherent detection (because of the root square dependence of the electrical signal on the Brillouin optical power), leading to a two-way sensing range improvement which is twice the coding gain (i.e. $\sim 15 \mathrm{~dB}$ ). This 


\section{OThU4.pdf}
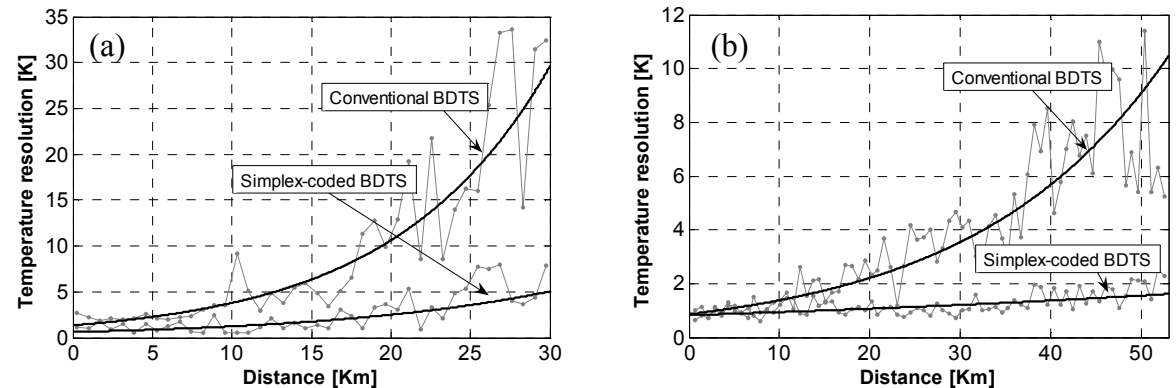

Fig. 2. Temperature resolution in LPR-based temperature sensor for both conventional and 127-bit Simplex coded BDTS (a) Based on direct detection. (b) Based on coherent detection.

feature is confirmed by the results of Fig. $2 b$, where we can observe that the same temperature resolution of $1.7 \mathrm{~K}$ is achieved at $14.5 \mathrm{~km}$ distance with conventional BDTS, and at $53 \mathrm{~km}$ with coding; hence, the sensing range of our conventional BDTS based on coherent detection can be enhanced by $\sim 38.5 \mathrm{~km}$ (i.e. $\sim 15 \mathrm{~dB}$ twoway) with the use of 127-bit Simplex coding. This represents twice the sensing range enhancement provided by coding in direct-detection schemes, resulting in a significant benefit of optical coding in the accuracy of coherent-detection based sensors.

\section{Simplex-coding applied to simultaneous strain and temperature sensing}

Using the experimental setup shown in Fig. 2b, both BFS and SpBS power have been measured in order to quantify the benefits provided by optical pulse coding in simultaneous strain and temperature sensing. Note that, the use of coding in coherent-detection based sensors also enhances the accuracy in BFS measurement, since the BFS $\left(\delta v_{B}\right)$ depends on the SNR according to the relation: $\delta v_{\mathrm{B}}=\Delta v_{\mathrm{B}} /(4 \cdot \mathrm{SNR})^{1 / 4}$ (where $\Delta v_{B}$ is the Brillouin light linewidth [7]). The above-mentioned features can then be exploited to improve the accuracy of simultaneous temperature and strain sensing based on both SpBS power and BFS measurements.

By calculating the rms noise in both BFS and SpBS power measurements, simultaneous strain and temperature resolution have been calculated through Eq. (2). Figs. 3a and $3 b$ show a comparison of the experimental temperature and strain resolutions obtained with both single-pulse and 127-bit Simplex coding, when measuring both physical variables simultaneously. A temperature resolution of $\sim 10 \mathrm{~K}$ is achieved at $53 \mathrm{~km}$ for the single-pulse case, which is improved to $\sim 1.6 \mathrm{~K}$ when using 127-bit Simplex coding. In Fig. $3 \mathrm{~b}$ we can see that, thanks to the SNR enhancement provided by coding the strain resolution at fiber-end $(53 \mathrm{~km})$ is improved from $\sim 250 \mu \varepsilon$ (obtained with single-pulses) down to $\sim 50 \mu \varepsilon$ when 127-bit Simplex coding is applied.
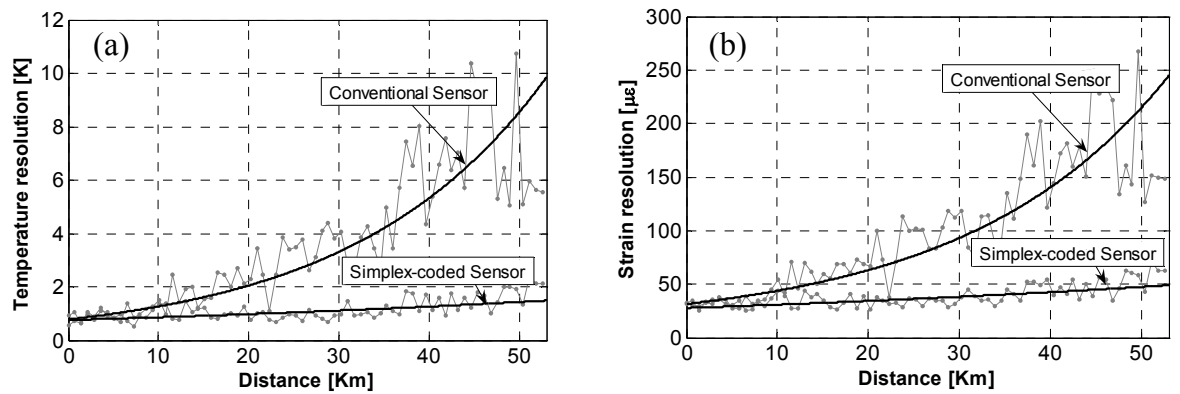

Fig. 3. (a) Temperature and (b) strain resolution vs distance, for both conventional and 127-bit Simplex-coded SpBS-based hybrid sensor.

In conclusion, the use of coding technique in hybrid Brillouin-based sensors allows for a notable enhancement in both strain and temperature resolutions when employing coherent detection in BFS and SpBS power measurements. This also leads to a significant sensing distance enhancement with the same temperature and strain resolutions of single-pulses case (nearly $40 \mathrm{~km}$ enhancement was achieved with 127-bit Simplex coding, equivalent to $\sim 15 \mathrm{~dB}$ two-way span budget). Thus, coding technique results in an effective solution to improve the performance of simultaneous strain and temperature measurements, avoiding the use of optical pulse amplification or high power laser sources.

\section{References}

[1] Nature Photonics, "Optical-fibre Sensors," Tech. Focus, vol. 2, pp. 143-158, 2008.

[2] B. Culshaw, "Optical fiber sensor technologies: opportunities and-perhaps-pitfalls," J. Lightwave Technol., vol. 22, no. 1, pp. 39-50, 2004.

[3] S. M. Maughan et al., "Simultaneous distributed fibre temperature and strain sensor using microwave coherent detection of spontaneous Brillouin backscatter," Meas. Sci. Technol., vol 12, pp 834-842, 2001.

[4] P. C. Wait, T. P. Newson., "Landau Placzek ratio applied to distributed fiber sensing," Opt. Commun., vol. 122, pp. 141-146, 1996.

[5] W. Jin et al., "Simultaneous measurement of strain and temperature: error analysis," Opt. Eng. 36(2), pp. 598-609 Feb. 1997.

[6] M. A. Soto et al., "Brillouin-based distributed temperature sensor employing pulse coding," IEEE Sens. J., vol. 8, n. 3, pp 225-226, 2008.

[7] T. Horiguchi et al., "Development of a Distributed Sensing Technique Using Brillouin Scattering," J. Lightwave Technol., vol. 13, no. 7, pp. 1296-1301, 1995. 